\title{
IDEAL-TRIANGULARIZABILITY AND COMMUTATORS OF CONSTANT SIGN
}

\author{
ROMAN DRNOVŠEK AND MARKO KANDIĆ
}

Abstract. Let $E$ be a Banach lattice with order continuous norm, and let $A$ and $B$ be positive compact operators such that the commutator $A B-B A$ is also positive. We prove that if $A$ and $B$ are ideal-triangularizable, then they are simultaneously ideal-triangularizable, or equivalently, the sum $A+B$ is ideal-triangularizable. We then show several related results for operators of constant sign (an operator $T$ on $E$ is of constant sign if either $T$ or $-T$ is positive). In particular, we consider ideal-triangularizability for Lie sets of compact operators of constant sign (a set of operators is a Lie set whenever it is closed under taking commutators).

Mathematics subject classification (2010): 46B42, 47A15, 47B65, 47B47.

Keywords and phrases: Banach lattices, positive operators, invariant subspaces, commutators.

\section{REFERENCES}

[1] Y. A. Abramovich, C. D. Aliprantis, An Invitation to Operator Theory, American Mathematical Society, Providence, 2002.

[2] C. D. Aliprantis, O. Burkinshaw, Positive operators, Springer, 2006.

[3] J. Bračič, R. Drnovšek, Y. B. Farforovskaya, E. L. Rabkin, J. Zemánek, On positive commutators, Positivity 14 (2010), 431-439.

[4] R. DRNOvŠEK, Common invariant subspaces for collections of operators, Integral Equat. Oper. Th. 39 (2001), 253-266.

[5] R. DrnovšEk, M. Jesenko, M. Kandić, Positive commutators and collections of operators, Operators and Matrices 6 (2012), 535-542.

[6] R. DRNOVŠEK, M. KANDIĆ, Ideal-triangularizability of semigroups of positive operators, Integral Equat. Oper. Th. 64 (2009), no. 4, 539-552.

[7] R. DrnovŠEK, M. Kandić, More on positive commutators, J. Math. Anal. Appl. 373 (2011), 580 584.

[8] R. DRnOvŠEK, M. KAndić, From local to global ideal-triangularizability, Linear Multilinear Algebra 62 (2014), no. 12, 1616-1628.

[9] R. DrnovšEk, D. Kokol-Bukovšek, L. Livshits, G. MacDonald, M. OMladič, H. RaDJAVI, An irreducible semigroup of non-negative square-zero operators, Integral Equat. Oper. Th. $\mathbf{4 2}$ (2002), no. 4, 449-460.

[10] N. GAO, Extensions of Perron-Frobenius theory, Positivity 17 (2013), no. 4, 965-977.

[11] M. KANDIĆ, Ideal-triangularizability of upward directed sets of positive operators, Ann. Funct. Anal. 2 (2011), no. 1, 206-219.

[12] M. KAndić, Multiplicative coordinate functionals and ideal-triangularizability, Positivity 17 (2013), no. 4, 1085-1099.

[13] M. Kennedy, Triangularization of a Jordan algebra of Schatten operators, Proc. Amer. Math. Soc. 136 (2008), 2521-2527.

[14] M. Kennedy, H. RADJAVI, Spectral conditions on Lie and Jordan algebras of compact operators, J. Funct. Anal. 256 (2009), no. 10, 3143-3157.

[15] M. Kennedy, V. S. Shulman, Y. V. TUROVS KiI, Invariant subspaces of subgraded Lie algebras of compact operators, Integral Equat. Oper. Th. 63 (2009), 47-93. 
[16] M. Konvalinka, Triangularizability of Polynomially Compact Operators, Integral Equat. Oper. Th. 52 (2005), 271-284.

[17] V. Lomonosov, Invariant subspaces for the family of operators commuting with compact operators, Funkcional. Anal. i Priložen. 7 (1973), 55-56 (Russian); Funct. Anal. and Appl. 7 (1973), 213-214 (English).

[18] W. A. J. Luxemburg, A. C. ZaAnen, Riesz spaces I, North Holland, Amsterdam, 1971.

[19] A. PietsCh, Eigenvalues and s-numbers, Cambridge Univ. Press, 1987.

[20] H. Radjavi, P. Rosenthal, Simultaneous Triangularization, Springer-Verlag, New York, 2000.

[21] H. H. SchAEFER, Banach lattices and positive operators, Springer-Verlag, Berlin-Heidelberg-New York, 1974.

[22] V. S. Shulman, Y. V. TuRovskit, Joint spectral radius, operator semigroups, and a problem of W. Wojtyński, J. Funct. Anal. 177 (2000), 383-441.

[23] V. S. Shulman, Y. V. TuRovs KiI, Invariant subspaces of operator Lie algebras and Lie algebras with compact adjoint action, J. Funct. Anal. 223 (2005), 425-508.

[24] Y. V. TUROVSKII, Spectral properties of certain Lie subalgebras and the spectral radius of subsets of a Banach algebra, in Spectral Theory of Operators and Its Applications, Vol. 6, pp. 144-181, Elm, Baku, 1985 (Russian).

[25] W. WOJTYŃSKI, Engel's theorem for nilpotent Lie algebras of Hilbert-Schmidt operators, Bull. Acad. Polon. Sci. 24 (9) (1976), 797-801. 